\title{
Synthesising and Implementing Tableau Calculi for Interrogative Epistemic Logics
}

\author{
Ştefan Minică ${ }^{1}$, \\ Mohammad Khodadadi, Renate A. Schmidt and Dmitry Tishkovsky ${ }^{2 *}$ \\ 1 Amstelveen, The Netherlands \\ stefan.minica@gmail.com \\ 2 The University of Manchester, Manchester, UK \\ khodadadi,dmitry, schmidt@cs.man.ac.uk
}

\begin{abstract}
This paper presents a labelled tableau approach for deciding interrogative-epistemic logics (IEL). Tableau calculi for these logics have been derived using a recently introduced tableau synthesis method. We also consider an extension of the framework for a setting with questioning modalities over sequences of formulae called sequential questioning logic (SQL). We have implemented the calculi using two approaches. The first implementation has been obtained with the tableau prover generation software METTEL2, while the other implementation is a prover implemented in Haskell.
\end{abstract}

\section{Introduction}

The paper focusses on developing and implementing automated reasoning tools for interrogative epistemic logics (IEL). Interrogative or erotetic logics have a long tradition alongside declarative and epistemic logics. Interrogative Epistemic Logic (henceforth, IEL) also referred to as DELQ (for Dynamic Epistemic Logic of Questions), enriches a standard multi-agent epistemic modal logic with interrogative components $\lfloor 15,7\rfloor$. Intuitively this is done by adding an "issue" relation over a set of possible worlds. This relation is meant to represent structural changes brought about by dynamic actions of raising and answering questions. Besides the standard epistemic modality the logic also introduces a static modality over the issue relation. This gives rise to interaction between the epistemic and interrogative components. Such aspects are captured by an intersection modality which is then used to describe how dynamic questioning effects depend on the structure of the issues raised and previous knowledge.

A second addition are the dynamic actions that express interrogative or epistemic events explicitly in the language. Their effect is to change the interrogative and epistemic states and to add more structure to the existing issue or epistemic relations.

While automated reasoning tools are widespread for declarative and epistemic modal logics, for interrogative epistemic logics there are currently no implemented automated reasoning systems. The usefulness of automating reasoning for other logics, such as epistemic modal logics, has been proven already by many applications. Very often in epistemic scenarios obtaining the relevant information is an essential part. Adding an interrogative component makes modelling and reasoning about obtaining relevant information possible.

For dynamic epistemic logics (DEL), automated reasoning tools focused so far on solving model-checking tasks 17]. Other tableau-based provers for modal logics, e.g., 3, incorporate dynamic modalities for informative actions, e.g., public announcements $\lfloor 4,2$. However, none

*This research is supported by UK EPSRC research grant EP/H043748/1. 
of the existing software tools contain questioning modalities and moreover, none of them offer a generic method to generate a prover for a logic starting from a semantic specifications.

Planning in contexts involving interaction between questions and knowledge is reducible to testing validities of interrogative epistemic logic. However, the existing proof systems for dynamic epistemic logics 44,14 are not fully automated. They are usually Hilbert-style calculi in which formulae with non factual content have special substitution rules.

A longstanding problem for dynamic logics is the fact that they are not closed under uniform substitution, and therefore, they are not suitable for an algebraic treatment and do not lend themselves well to automatic reasoning techniques. Previous research in this area focused on identifying substitution closed fragments of such logics, which can still preserve some of the features that have made dynamic logics so successful for modelling information exchange.

The approach of this paper gives an alternative solution based on first translating the semantics of the modal language into many-sorted first-order logic and then turning it into a tableau calculus for the corresponding first-order fragment. Based on this tableau calculus two tableau based reasoning tools have been developed for interrogative-epistemic logics.

The paper is structured as follows. We start in Section 2 by introducing the details of IEL. Then we apply the tableau synthesis framework to IEL in Section 3. In Section 4 we present an extended logic, called SQL, which uses sequences of formulae inside the dynamic modalities. We continue in Section 5 with introducing and discussing the MeTTeL implementation for IEL. In Section 6 we present and discuss the Haskell implementation Qtab. Ihs for IEL which also illustrates the extension to questioning sequences. We draw some conclusions in the final section. Further implementation details and illustrative code output, which could not be included due to space limitations, can be found in the long version of the paper $[8$.

\section{Interrogative Epistemic Logics}

The approach of IEL $\lfloor 15,7$. starts by enriching a standard multi-agent epistemic modal logic with interrogative components. This is done in two stages. The first addition consists of a static modality over an "issue" relation. The intuitive meaning of this modality is close to the traditional epistemic notion, but instead of representing actual knowledge it stands for what the agents would like to find out. It represents future epistemic goals that are expressed by asking questions and will eventually be achieved by obtaining answers.

For technical reasons a third modality, expressing the interaction between the epistemic and the interrogative components is also introduced using the intersection of the standard epistemic relation and the newly introduce issue relation. This also has an intuitive meaning that goes beyond the traditional epistemic notion. It expresses how the future knowledge depends on both the current epistemic state of the agent and the epistemic goals guiding the ongoing questioning dynamics. This is what the agent will come to know if all the questions he raised so far would be answered one way or another.

In this paper nominals are added to the language alongside propositions. The second addition consists of two dynamic modalities, one for questioning actions or queries and one for answering actions or resolution actions. Intuitively, such modalities change the underlying structures by refining their component relations.

A formula $\varphi$ in the language of IEL is defined by the following BNF:

$$
\varphi \quad::=n|p| \neg \varphi|\varphi \vee \varphi| \square \varphi \mid[Q] \varphi
$$

Here, $n, p, a$ denoting nominals, propositions, and agent labels, respectively. $\square$ stands for modalities, that is $\square \in\left\{Q_{a}, X_{a}, K_{a}\right\}$, respectively being static questioning, interaction, and 
epistemic modalities. Finally, $Q$ stands for actions that change the underlying models, that is $Q \in\left\{\varphi_{a}, !_{a}\right\}$, representing dynamic questioning actions and resolution actions, respectively. This language is meant to express minimal interrogative-epistemic facts. The @ operator, which is a standard addition for hybrid logics is introduced later in the specification language where it is useful for both internalizing the semantics and formulating labeled tableau rules.

The language can express the interaction between questions and information in two ways. First, by using a (static) intersection modality $X_{a} \varphi$. Second, through the dynamic modalities $[Q]$, encoding model-changing operations by means of questioning and resolution actions.

The logic has a standard modal semantics over issue-epistemic models, $M=\langle W, \stackrel{a}{\approx}, \stackrel{a}{\sim}, V\rangle$. When used inside a tuple representing a model $\stackrel{a}{\approx}$ and $\stackrel{a}{\sim}$ are meant as shorthand notations for $(\stackrel{a}{\approx})_{a \in A}$ respectively $(\stackrel{a}{\sim})_{a \in A}$ for $A$ the set of all agent labels. We use the expected Boolean clauses and the usual relational (modal) clauses involving $\underset{\sim}{\approx}$ for $Q_{a}$ and $\stackrel{a}{\sim}$ for $K_{a}$. We also use equivalence relations for $\approx$ and $\sim$ throughout the paper. However, if needed, the framework can be generalized to other structures by correspondingly changing the background theory.

The intersection modality $X_{a}$ is defined using $\stackrel{a}{\approx} \cap \stackrel{a}{\sim}$ as follows:

$$
M \models_{w} X_{a} \varphi \quad \text { iff } \quad \forall v \in W: w(\stackrel{a}{\approx} \cap \stackrel{a}{\sim}) v \Rightarrow M \models_{v} \varphi
$$

Dynamic modalities express model changing operations of asking and resolution:

$[\varphi ?]_{a} \psi \quad$ "after $\varphi$ is asked, $\psi$ is the case" $\quad M_{?}=\langle W, \stackrel{a}{\approx}, \stackrel{a}{\sim}, V\rangle ; \quad \stackrel{a}{\approx}_{?}=\stackrel{a}{\approx} \cap \stackrel{\varphi}{=}_{M}$

$[!]_{a} \varphi \quad$ "after having answered the questions raised, $\varphi$ is true"

$$
M_{!}=\langle W, \stackrel{a}{\approx}, \stackrel{a}{\sim} !, V\rangle ; \quad \stackrel{a}{\sim} !=\stackrel{a}{\sim} \cap \stackrel{a}{\approx}
$$

Here, $\stackrel{\underline{\underline{\varphi}}}{=}_{M}=\left\{(w, v) \mid\|\varphi\|_{w}^{M}=\|\varphi\|_{v}^{M}\right\}$ is the set of $M$-world pairs in which $\varphi$ has the same truth value. The intuitive reading for a questioning action represented by the modality $[\varphi ?]_{a} \psi$ is that of splitting the domain into $\varphi$ worlds and non- $\varphi$ worlds, by raising a question, or by making $\varphi$ an issue. The intuitive reading for the resolution action represented by the $[!]_{a} \varphi$ modality is to add new knowledge by refining the epistemic relation in such a way that afterwards all the issues raised so far are solved. Indexing the actions with agent labels can also model privacy in questioning or can be used to add agent-specific preconditions for question execution. However, we assume our actions to be 'public' and 'preconditionless', i.e., they affect the epistemic/questioning states for all agents, and they do not require any conditions for execution. For this reason, indexing the modalities with agent labels will only play a genuine role in this paper for the static part of the logic.

The language of IEL has two parts. The static part is a hybrid modal logic with nominals and intersection. The dynamic part adds the dynamic modalities capturing model changing operations. The static part of the logic is axiomatised by a customary hybrid logic system 12 . with nominals, S5 axioms for $\sim$ and $\approx$, and an intersection axiom for static resolution expressed by the following pure formula:

$$
\widehat{K}_{a} i \wedge \widehat{Q}_{a} i \leftrightarrow \widehat{X}_{a} i, \quad \text { where } i \text { is a nominal. }
$$

Here, $\widehat{Q}_{a}, \widehat{K}_{a}$ and $\widehat{X}_{a}$ are the diamond modalities defined as the duals of the box modalities $Q_{a}, K_{a}, X_{a}$ introduced before.

The dynamic part of IEL introduces modalities which change the underlying static models. The logical behaviour of this new kind of connectives is captured by reduction axioms. These describe the relation between the underlying static structures before and after a questioning 
action or resolution action takes place. Formulas containing dynamic modalities can be reduced to equivalent static formulas using reduction axioms like the following ones (for $b \in\{n, p\}$, $\square \in\{Q, X\}$ and $q \in\{\varphi ?, !\})$ :

$$
\begin{aligned}
& {[q]_{a} b \leftrightarrow b, \quad[q]_{a} \neg \psi \leftrightarrow \neg[q]_{a} \psi, \quad[q]_{a}(\psi \wedge \chi) \leftrightarrow[q]_{a} \psi \wedge[q]_{a} \chi} \\
& {[!]_{a} \square_{a} \psi \leftrightarrow \square_{a}[!]_{a} \psi, \quad[!]_{a} K_{a} \varphi \leftrightarrow X_{a}[!]_{a} \varphi, \quad[\varphi ?]_{a} K_{a} \psi \leftrightarrow K_{a}[\varphi ?]_{a} \psi} \\
& {[\varphi ?]_{a} Q_{a} \psi \leftrightarrow\left(\varphi \wedge Q_{a}\left(\varphi \rightarrow[\varphi ?]_{a} \psi\right)\right) \vee\left(\neg \varphi \wedge Q_{a}\left(\neg \varphi \rightarrow[\varphi ?]_{a} \psi\right)\right)} \\
& {[\varphi ?]_{a} X_{a} \psi \leftrightarrow\left(\varphi \wedge X_{a}\left(\varphi \rightarrow[\varphi ?]_{a} \psi\right)\right) \vee\left(\neg \varphi \wedge X_{a}\left(\neg \varphi \rightarrow[\varphi ?]_{a} \psi\right)\right)}
\end{aligned}
$$

This treatment is in line with the generic DEL methodology introduced in $[4,14$ extended to include an additional interrogative component. Further technical details, possible extensions and examples of applications of IEL can be found in $[7,15]$.

We start from IEL as minimal logic when synthesizing and implementing the tableau calculus. Several extensions of the framework that handle various levels of privacy for epistemicquestioning actions can be added in a modular way using the same general synthesis method.

- Multi-agent questioning preconditions

- Group-opaque dynamic questioning effects

- Epistemic indistinguishability in questioning

- Dynamic questioning sequences

Due to lack of space in this paper we will discuss in detail only the last extension.

The extension requires questioning actions of a more complex type capable to model modalities over sequences of questions not just one formula. We briefly motivate now why such an extension is desirable and useful. The concrete details of the extension method are introduced later in Section 4, after all the needed details of the specification language are introduced.

Note that the standard IEL reduction axioms do not have cases for iterated modalities. Indeed, such cases are not, at some level of abstraction, necessary since they can be dealt with logically "from inside out". For any formulae $\varphi, \psi, \chi$ of IEL, $[\varphi$ ?] $[\psi$ ?] $\chi$ can be dealt with in the following order, given the recursive structure of the reduction axioms:

$$
[\varphi ?][\psi ?] \chi \leftrightarrow[\varphi ?](\operatorname{trs}([\psi ?] \chi)) \leftrightarrow \operatorname{trs}([\varphi ?](\operatorname{trs}([\psi ?] \chi)))
$$

Here trs stands for the translation of the right side in the reduction axioms from Equation 1, as defined in Equation 4. While such a rule of thumb can be useful to some extent for human reasoning it is nevertheless not optimal for automatic reasoning. Even though we left out iterated modalities during the exposition of the logic we later deal with them as the implementation details require them. For this a direct recursion over the formulae would be optimal, and we approach this aspect in both of our implementations in Sections 5 and 6 . Here we only briefly discuss some of the available modelling options.

One possible way to achieve this is by directly reducing iterated modalities to an equivalent non-iterated dynamic modality and then use the existing reduction axioms. For instance, for public announcement logic (PAL), which uses world-elimination instead of link-cutting, iteration of dynamic modalities boils down to the following equivalence for announcement composition:

$$
[! \varphi][! \psi] \chi \leftrightarrow[!(\varphi \wedge[! \varphi] \psi)] \chi
$$

However, there can be no such nor similar equivalent for IEL without sequences:

No single question can induce a 4-equivalence-classes partition. 
All these complications are avoided by a language with questioning sequences:

$$
[\varphi ?][\psi ?] \chi \leftrightarrow[\langle\varphi, \psi\rangle ?] \chi
$$

This can be achieved in a modular way by keeping the syntax and the semantics of the language unchanged for both the static part and the dynamic resolution part and replacing our initial questioning modality with a modality defined over sequences of questions. This is also a dynamic modality for the collective action of asking questions or raising issues but the syntax uses a list of formulae $\sigma=\left\langle\varphi_{0}, \ldots, \varphi_{n}\right\rangle$ :

$$
[\sigma ?] \varphi \text { "after the questions in } \sigma \text { are asked, } \varphi \text { is the case". }
$$

The semantic definition of the dynamic modality is changed accordingly, the action's effect is to change an initial model $M$ into a new model $M_{\sigma ?}=\langle W, \underset{\sim}{\approx}, \stackrel{a}{\sim}, V\rangle$ with:

$$
\stackrel{x}{\approx}_{?}=\stackrel{x}{\approx}_{\langle\rangle ?} \cap \bigcap_{n=0}^{|\sigma|-1}{\stackrel{\varphi_{n}}{=}}_{M_{n}} \text { for any agent } x .
$$

For any model $M$ and questioning sequence $\sigma=\left\langle\varphi_{0}, \ldots, \varphi_{n}\right\rangle$, the model $M_{k}$ denotes the model obtained after a questioning action using the sequence $\sigma_{k}=\left\langle\varphi_{0}, \ldots, \varphi_{k}\right\rangle$ for $0 \leq k \leq n$. An empty questioning sequence does not change a model: $M_{\langle\rangle ?}=M$ and, in particular, $\approx_{\langle\rangle ?}=\approx$. This allows one to deal with longer sequences recursively using a head-tail pattern:

$$
[\langle\rangle ?] \varphi \leftrightarrow \varphi \text { and }\left[\left\langle\varphi_{0}, \varphi_{1}, \ldots, \varphi_{n}\right\rangle ?\right] \varphi \leftrightarrow\left[\left\langle\varphi_{0}\right\rangle ?\right]\left[\left\langle\varphi_{1}, \ldots, \varphi_{n}\right\rangle ?\right] \varphi .
$$

The case of iterating the resolution modality [!] is much simpler because sequences of any length $[\langle!, !, \ldots\rangle]$ can be collapsed to a resolution sequence of length one $[\langle!\rangle]$. This is so because the resolution modality is idempotent: !;! = !. Therefore, we only have to consider iteration between sequential asking modalities and single, i.e., depth one, resolution modalities.

This leads to the more general reduction axioms we introduce in Section 4. The reduction axioms in Equation 1 can be also seen as particular cases in which we take $n=1$ inside the dynamic questioning modality $[\sigma(n)$ ?]. We lift the restriction to single questions from the vanilla version of the language and allow questioning sequences in two stages. First by introducing special reduction axioms for minimal sequences, i.e., sequences of length two, in Section 5. Second, we introduce questioning sequences of arbitrary length and give fully general reduction axioms for them in Sections 4 and 6. We continue our exposition using the simplest version of IEL and afterwards return in Section 4 to considering the SQL extension in more detail.

\section{The Tableau Synthesis Framework Applied to IEL}

In order to obtain a sound, complete and terminating tableau calculus for IEL we apply the tableau synthesis framework introduced in $[11,10$. In brief, the tableau synthesis method works as follows. The user defines the formal semantics of their logic in the first-order specification language of the framework. The semantic specification can then be automatically transformed into tableau rules that form a calculus which is sound and complete provided the semantic specification satisfies certain conditions. In a next step the possibility to refine the tableau calculus in two ways is explored. First, it may be possible to refine that tableau rules by reducing their branching factor and, second, it may be possible to internalise semantic constructs of the tableau language in the language of the logic. Finally, the unrestricted blocking mechanism 
can be added to the obtained calculus. The blocking mechanism ensures termination of the calculus if the logic has the finite model property. The final calculus is sound, complete and terminating, and, hence, provides the basis for a decision procedure for the logic.

The object language of specification of syntax of the logic IEL has several distinct sorts. The main sort of the language is the sort of formulae (sort $\mathrm{f}$ ) which are denoted as $\varphi, \psi, \ldots$. Other sorts are individuals (sort i) denoted $i, j, \ldots$, propositional atoms ( $\operatorname{sort} \mathrm{p}$ ) denoted $p, q, \ldots$, and agent labels (sort a) denoted as $a, a_{0}, a_{1}, \ldots$.

For reasons of economy and simplicity, we fix a (minimal) set of connectives for the syntax specification of IEL. The connectives and their types are listed in Figure 1

Useful additions to the specification language include the singleton set operator $\{\cdot\}$ and the operator \#., which respectively link the individual and formula sorts, and the proposition and formula sorts. The operator @. is the at (or satisfaction) operator, which is useful for internalising the semantic specification.

The meta-language of IEL for the specification of the semantics extends the object language of IEL with an additional domain sort $\mathrm{d}$ and the following symbols: binary predicate symbols (of type $(\mathrm{d}, \mathrm{d})$ ) $R_{\underset{\sim}{\sim}}, R_{\underset{\sim}{\approx} \cap \stackrel{a}{\sim}}$, and $R_{a} ;$ the equality symbol $\dot{\sim}$ (we use a dot to distinguish equality from the issue relation); domain variables $x, y, z, \ldots$; and the first-order quantifiers $\forall$ and $\exists$. Finally, the meta-language contains three interpretation symbols $\nu_{\mathrm{i}}, \nu_{\mathrm{f}}$, and $\nu_{\mathrm{p}}$. For every nominal $i$ of sort $\mathbf{i}, \nu_{\mathrm{i}}(i)$ is a term of sort $\mathrm{d}$. For every IEL-formula $\varphi$ of sort $\mathbf{f}$, proposition $p$ of sort $\mathrm{p}$, and term $t$ of sort $\mathrm{d}, \nu_{\mathrm{f}}(\varphi, t)$ and $\nu_{\mathrm{p}}(p, t)$ are atomic formulae in the semantic specification language for IEL.

Figure 2 shows the definition of the semantics of the IEL connectives in the meta-language. We give the standard Boolean and modal semantic definitions in the right column and the semantics of the sort-bridging connectives in the left column. Both are followed by definitions for the dynamic modalities. We denote the set of all these formulae by $S_{0}$.

Additionally there are conditions which specify properties of relations and equality. They are captured by the background theory axioms $S^{b}$ which are listed in Figures 3 and 4

The described semantic specification captures in first-order sentences the semantic conditions for IEL from Section 2. In particular, the difference between the semantic definition of the static modalities and the dynamic modalities becomes more apparent. While the static modalities are dropped by the definitions, the dynamic ones are only dropped in the definitions for atomic components in their scope. However, for complex formulae the dynamic modality is applied in the right hand side of the definition to a formula with lower complexity. This is also reflected in the semantic specifications that the reduction axioms vary depending on whether they are for propositional atoms, for singletons, and for formulae.

The next step is to transform the semantic specification into a normalised implicational form $\left[11\right.$. This is done by decomposing each logical equivalence of the specification $S_{0}$ into the left-to-right implication and the contrapositive of the right-to-left implication. The resulting sets of formulae are denoted by $S^{+}$and $S^{-}$.

\begin{tabular}{c|c} 
Connective & Type \\
\hline$\{\cdot\}$ & $\mathrm{i} \mapsto \mathrm{f}$ \\
$@$. & $(\mathrm{i}, \mathrm{f}) \mapsto \mathrm{f}$ \\
$\neg \cdot$ & $\mathrm{f} \mapsto \mathrm{f}$ \\
$\cdot \wedge \cdot$ & $(\mathrm{f}, \mathrm{f}) \mapsto \mathrm{f}$ \\
\hline
\end{tabular}

\begin{tabular}{c|c} 
Connective & Type \\
\hline$\# \cdot$ & $\mathrm{p} \mapsto \mathrm{f}$ \\
$Q$. & $(\mathrm{a}, \mathrm{f}) \mapsto \mathrm{f}$ \\
$K \cdot$ & $(\mathrm{a}, \mathrm{f}) \mapsto \mathrm{f}$ \\
$X$. & $(\mathrm{a}, \mathrm{f}) \mapsto \mathrm{f}$ \\
\hline
\end{tabular}

\begin{tabular}{c|c} 
Connective & Type \\
\hline$[\cdot ?] \cdot \cdot$ & $(\mathrm{f}, \mathrm{a}, \mathrm{f}) \mapsto \mathrm{f}$ \\
{$[!] \cdot \cdot$} & $(\mathrm{a}, \mathrm{f}) \mapsto \mathrm{f}$ \\
& \\
\hline
\end{tabular}

Figure 1: Connectives of the object language of IEL. 
Tableau Synthesis for Interrogative Epistemic Logics S. Minică, M. Khodadadi, R. A. Schmidt, D. Tishkovsky

$$
\begin{aligned}
& \forall x\left(\nu_{\mathrm{f}}(\{i\}, x) \leftrightarrow x \dot{\approx} \nu_{\mathrm{i}}(i)\right) \\
& \forall x\left(\nu_{\mathrm{f}}(\# p, x) \leftrightarrow \nu_{\mathrm{p}}(p, x)\right. \\
& \forall x\left(\nu_{\mathrm{f}}\left(@_{i} \varphi, x\right) \leftrightarrow \nu_{\mathrm{f}}\left(\varphi, \nu_{\mathrm{i}}(i)\right)\right) \\
& \left.\forall x\left(\nu_{\mathrm{f}}\left([\varphi ?]_{a} \# p, x\right) \leftrightarrow \nu_{\mathrm{f}}(\# p, x)\right)\right) \\
& \left.\forall x\left(\nu_{\mathfrak{f}}\left([q]_{a}\{i\}, x\right) \leftrightarrow \nu_{\mathfrak{f}}(\{i\}, x)\right)\right) \\
& \left.\forall x\left(\nu_{\mathrm{f}}\left([q]_{a} \neg \psi, x\right) \leftrightarrow \nu_{\mathrm{f}}\left(\neg[q]_{a} \psi, x\right)\right)\right) \\
& \forall x\left(\nu_{\mathrm{f}}\left([\varphi ?]_{a} K_{a} \psi, x\right) \leftrightarrow \nu_{\mathrm{f}}\left(K_{a}[\varphi ?] \psi, x\right)\right) \\
& \forall x\left(\nu_{\mathrm{f}}\left([!]_{a} K_{a} \psi, x\right) \leftrightarrow \nu_{\mathrm{f}}\left(X_{a}[!]_{a} \psi, x\right)\right) \\
& \forall x\left(\nu_{\mathrm{f}}\left([\varphi ?]_{a} Q_{a} \psi, x\right) \leftrightarrow\left(\nu_{\mathrm{f}}\left(\varphi \wedge Q_{a}\left(\neg \varphi \vee[\varphi ?]_{a} \psi\right), x\right) \vee \nu_{\mathrm{f}}\left(\neg \varphi \wedge Q_{a}\left(\varphi \vee[\varphi ?]_{a} \psi\right), x\right)\right)\right. \\
& \forall x\left(\nu_{\mathrm{f}}\left([\varphi ?]_{a} X_{a} \psi, x\right) \leftrightarrow\left(\nu_{\mathrm{f}}\left(\varphi \wedge X_{a}\left(\neg \varphi \vee[\varphi ?]_{a} \psi\right), x\right) \vee \nu_{\mathrm{f}}\left(\neg \varphi \wedge X_{a}\left(\varphi \vee[\varphi ?]_{a} \psi\right), x\right)\right)\right. \\
& \forall x\left(\nu_{\mathrm{f}}(\neg \varphi, x) \leftrightarrow \neg \nu_{\mathrm{f}}(\varphi, x)\right) \\
& \left.\forall x\left(\nu_{\mathrm{f}}(\varphi \wedge \psi, x) \leftrightarrow \nu_{\mathrm{f}}(\varphi, x) \wedge \nu_{\mathrm{f}}(\psi, x)\right)\right) \\
& \forall x\left(\nu_{\mathrm{f}}\left(Q_{a} \varphi, x\right) \leftrightarrow \forall y\left(R_{\underset{\sim}{a}}(x, y) \rightarrow \nu_{\mathrm{f}}(\varphi, y)\right)\right) \\
& \forall x\left(\nu_{\mathrm{f}}\left(K_{a} \varphi, x\right) \leftrightarrow \forall y\left(R_{a}(x, y) \rightarrow \nu_{\mathrm{f}}(\varphi, y)\right)\right) \\
& \left.\forall x\left(\nu_{\mathrm{f}}\left(X_{a} \varphi, x\right) \leftrightarrow \forall y\left(R_{\underset{\sim}{a} \cap \underset{\sim}{\sim}}(x, y) \rightarrow \nu_{\mathrm{f}}(\varphi, y)\right)\right)\right) \\
& \forall x\left(\nu_{\mathrm{f}}\left([q]_{a}(\psi \wedge \chi), x\right) \leftrightarrow \nu_{\mathrm{f}}\left([q]_{a} \psi, x\right) \wedge \nu_{\mathrm{f}}\left([q]_{a} \chi, x\right)\right) \\
& \forall x\left(\nu_{\mathrm{f}}\left([!]_{a} Q_{a} \psi, x\right) \leftrightarrow \nu_{\mathrm{f}}\left(Q_{a}[!]_{a} \psi, x\right)\right) \\
& \forall x\left(\nu_{\mathrm{f}}\left([!]_{a} X_{a} \psi, x\right) \leftrightarrow \nu_{\mathrm{f}}\left(X_{a}[!]_{a} \psi, x\right)\right)
\end{aligned}
$$

Figure 2: Semantic specification $S_{0}$ of connectives for IEL $(q \in[\varphi ?],[!])$

$$
\begin{aligned}
& \forall x \forall y\left(R_{\underset{a}{\approx} \cap \underset{\sim}{\sim}}(x, y) \leftrightarrow R_{\underset{a}{a}}(x, y) \wedge R_{\underset{\sim}{\sim}}(x, y)\right), \\
& \forall x \forall y \forall z\left(\left(R_{\underset{\sim}{a}}(x, y) \wedge R_{\underset{\approx}{a}}^{\underset{(}{*}}(y, z)\right) \rightarrow R_{\underset{\sim}{a}}(x, z)\right), \quad \forall x \forall y \forall z\left(\left(R_{a}(x, y) \wedge R_{a}(y, z)\right) \rightarrow R_{\underset{\sim}{a}}(x, z)\right), \\
& \forall x \forall y \forall z\left(\left(R_{\underset{a}{\sim} \cap \underset{a}{\sim}}^{\sim}(x, y) \wedge R_{a} \cap \underset{\sim}{\sim}(y, z)\right) \rightarrow R_{a} \underset{\sim}{\sim}(x, z)\right), \\
& \forall x \forall y\left(R_{\underset{a}{\approx}}(x, y) \rightarrow R_{\underset{a}{\approx}}(y, x)\right), \quad \forall x \forall y\left(R_{\underset{a}{\approx} \cap \underset{\sim}{\sim}}(x, y) \rightarrow R_{\underset{\sim}{\approx} \cap \underset{a}{\sim}}(y, x)\right), \\
& \forall x \forall y\left(\underset{\sim}{R_{a}^{a}}(x, y) \rightarrow R_{\underset{\sim}{a}}^{\sim}(y, x)\right), \quad \forall x R_{\underset{a}{a}}(x, x), \quad \forall x R_{\underset{\sim}{\approx} \cap \underset{\sim}{\sim}}(x, x), \forall x R_{a}(x, x)
\end{aligned}
$$

Figure 3: Semantic specification of background theory axioms for the relations

It is not difficult to check that the semantic specification is well-defined in the sense of $\lfloor 11$. A semantic specification $S$ is well defined iff $S$ is normalized and the following conditions hold:

(wd1) $\forall S^{0}, \forall S^{b} \models \forall S$,

(wd2) the relation $\prec$ induced by $S$ is a well-founded ordering on formulae, and

(wd3) for every formula $\varphi=\sigma\left(\varphi_{1}, \ldots, \varphi_{m}\right)$, defining a connective $\sigma$ :

$$
\forall S^{0}, \forall S^{b} \uparrow \operatorname{sub}_{\prec}(\varphi) \models_{c} \forall \bar{x}\left(\left(\bigwedge \Phi_{+}^{\varphi} \rightarrow \phi^{\sigma}\left(\varphi_{1}, \ldots, \varphi_{m}, \bar{x}\right)\right) \wedge\left(\phi^{\sigma}\left(\varphi_{1}, \ldots, \varphi_{m}, \bar{x}\right) \rightarrow \bigvee \Phi_{-}^{\varphi}\right)\right)
$$

Here $\Phi_{+}^{\varphi}$ is the set obtained by collecting all instantiations of consequents from $S^{+}$(the positive specifications in $S$ ) matching the formula $\varphi . \Phi_{-}^{\varphi}$ is the set obtained by collecting all instantiations of antecedents from $S^{-}$(the negative specifications in $S$ ) matching formula $\varphi$.

Condition (wd1) expresses the decomposition of the specification $S$ to $S^{0}$ and $S^{b}$ after normalization. The set $S^{0}$ contains the connective definitions, and the set $S^{b}$ contains the background theory conditions. The set $S^{0}$ is further decomposed in two disjoint sets $S^{+}$and $S^{-}$. Since the semantic specification is the union of the connective definitions and the background

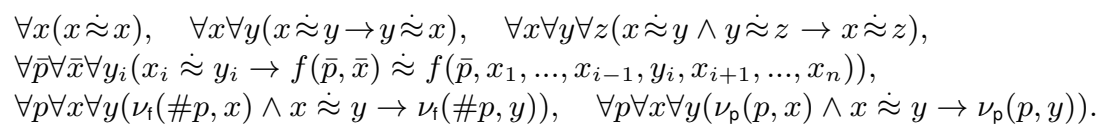

Figure 4: Semantic specification of equality axioms 
Tableau Synthesis for Interrogative Epistemic Logics S. Minică, M. Khodadadi, R. A. Schmidt, D. Tishkovsky

Tableau Expansion Rules (generated from $S_{0}=S^{+} \cup S^{-}$):

$$
\begin{aligned}
& \frac{@_{l}[\varphi ?] \square_{a} \psi}{\varliminf_{l} \varphi, @_{l} \square_{a}(\neg \varphi \vee[\varphi ?] \psi) \mid @_{l} \neg \varphi, @_{l} \square_{a}(\varphi \vee[\varphi ?] \psi)}(\square \in Q, X), \quad \frac{\varliminf_{l}[!] K_{a} \psi}{\varliminf_{l} X_{a}[!] \psi}, \\
& \frac{@_{l} \neg[\varphi ?] \square_{a} \psi}{@_{l}\left(\neg \varphi \vee \neg \square_{a}(\neg \varphi \vee[\varphi ?] \psi)\right), @_{l}\left(\varphi \vee \neg \square_{a}(\varphi \vee[\varphi ?] \psi)\right)}(\square \in Q, X), \quad \frac{@_{l} \neg[!] K_{a} \psi}{@_{l} \neg X_{a}[!] \psi} .
\end{aligned}
$$

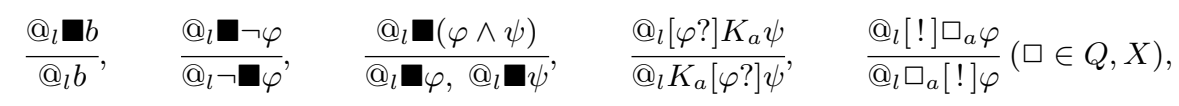

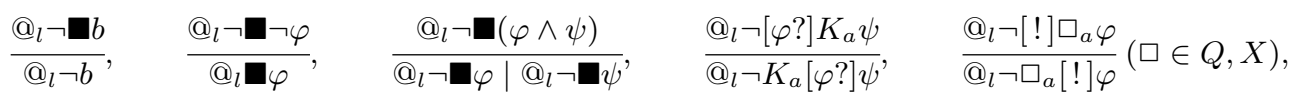

$$
\begin{aligned}
& \frac{@_{l} \neg \square_{a} \varphi}{@_{l} \diamond_{a}\left\{f_{\neg \square}(l, a, \varphi)\right\}, @_{f_{\neg \square}(l, a, \varphi) \neg \varphi}}(\square \in Q, K, X), \quad \frac{@_{l} \square_{a} \varphi, @_{l} \diamond_{a}\left\{l_{2}\right\}}{@_{l_{2}} \varphi}(\square \in Q, K, X), \\
& \frac{@_{l} \neg \neg \varphi}{@_{l} \varphi}, \quad \frac{@_{l} \varphi \wedge \psi}{@_{l} \varphi, @_{l} \psi}, \quad \frac{@_{l} \neg(\varphi \wedge \psi)}{@_{l} \neg \varphi \mid @_{l} \neg \psi},
\end{aligned}
$$

Background Theory Rules (generated from $S_{b}$ ):

$$
\begin{array}{rrr}
\frac{@_{l} \widehat{X}_{a}\left\{l_{2}\right\}}{@_{l} \widehat{Q}_{a}\left\{l_{2}\right\},}, & @_{l} \widehat{K}_{a}\left\{l_{2}\right\} & \frac{@_{l} \widehat{Q}_{a}\left\{l_{2}\right\}, @_{l} \widehat{K}_{a}\left\{l_{2}\right\}}{@_{l} \widehat{X}_{a}\left\{l_{2}\right\}}, \\
\frac{@_{l} \diamond_{a}\left\{l_{2}\right\}, @_{l_{2}}\left\{l_{3}\right\}}{@_{l} \diamond_{a}\left\{l_{3}\right\}}, & \frac{@_{l}\{l\}}{@_{l} \diamond_{a}\{l\}}, \frac{@_{l} \diamond_{a}\left\{l_{2}\right\}}{@_{l_{2}} \diamond_{a}\{l\}}, & \frac{@_{l} \diamond_{a}\left\{l_{2}\right\}, @_{l_{2}} \diamond_{a}\left\{l_{3}\right\}}{@_{l} \diamond_{a}\left\{l_{3}\right\}}, \\
\frac{@_{l} \diamond_{a}\left\{l_{2}\right\}}{@_{l_{2}}\left\{l_{2}\right\}}, \frac{@_{l}\left\{l_{2}\right\}}{@_{l_{2}}\{l\}}, \frac{@_{l} \neg\left\{l_{2}\right\}}{@_{l_{2}} \neg\{l\}}, & \frac{@_{l} \varphi}{@_{l}\{l\}}, \frac{@_{l} \varphi, @_{l}\left\{l_{2}\right\}}{@_{l_{2}} \varphi}, & \text { (Clash): } \frac{@_{l} \varphi, @_{l} \neg \varphi}{\perp} .
\end{array}
$$

Figure 5: Refined calculus for IEL, where $\mathbf{\square} \in\left\{[\varphi ?]_{a},[!]_{a}\right\}, b \in\{\# p,\{n\}\}, \diamond \in\{\widehat{Q}, \widehat{K}, \widehat{X}\}$

theory, conditions ( $\mathrm{wd} 1)$ and $(\mathrm{wd} 3)$ are trivially satisfied. Showing condition (wd2), i.e., wellfoundedness of the ordering $\prec$ induced by the normalised specification, is more involved than usual because of the statements capturing the reduction axioms. Well-foundedness of the order can be established by assigning IEL formulae the following complexity measure:

$$
\begin{aligned}
& c(p)=1, \quad c(!)=1, \quad c(\neg \varphi)=1+c(\varphi), \quad c(\varphi \wedge \psi)=1+\max (c(\varphi), c(\psi)), \\
& c\left(\square_{a} \varphi\right)=1+c(\varphi) \quad \text { for } \square_{a} \in\left\{K_{a}, Q_{a}, X_{a}\right\}, \text { and } \\
& c([q] \psi)=(c(q)+5) \cdot c(\psi) \text { for } q \in\{\varphi ?, !\} .
\end{aligned}
$$

Turning the normalised semantic specification into tableau rules in accordance with $\lfloor 11$. then produces a sound and complete tableau calculus for checking satisfiability for IEL. We do not present this calculus here, but present (in Figure 5) immediately the calculus obtained after refinement.

Two refinements described in $\lfloor 11\rfloor$ have been applied. The first refinement is the internalisation of the domain symbols including interpretation symbols $\nu_{\mathrm{i}}, \nu_{\mathrm{f}}$, and $\nu_{\mathrm{p}}$ in the language of the logic. For example, the rules generated for the $\square$ operators are $(\square \in\{Q, K, X\})$ :

$$
\frac{\nu_{\mathrm{f}}\left(\neg \square_{a} \varphi, l\right)}{R\left(l, f_{\neg \square}(l, a, \varphi)\right), \nu_{\mathrm{f}}\left(\neg \varphi, f_{\neg \square}(l, a, \varphi)\right)} \quad \text { and } \quad \frac{\nu_{\mathrm{f}}\left(\square_{a} \varphi, l\right), l_{2} \dot{\approx} l_{2}}{\neg R\left(l, l_{2}\right) \mid \nu_{\mathrm{f}}\left(\varphi, l_{2}\right)}
$$


$R$ denotes the appropriate accessibility relation associated with $\square_{a} . f_{\neg \square}$ represents one of three fixed Skolem functions used as a convenient way to create witnesses for formulae of existential extent. Because IEL is a hybrid logic it fully supports individuals and the rules can be rewritten as

$$
\frac{@_{l} \neg \square_{a} \varphi}{@_{l} \diamond_{a}\left\{f_{\neg \square}(l, a, \varphi)\right\}, @_{f_{\neg \square}(l, a, \varphi) \neg \varphi}} \text { and } \frac{@_{l} \square_{a} \varphi, @_{l_{a}} \diamond_{a}\left\{l_{2}\right\}}{@_{l} \neg \diamond_{a}\left\{l_{2}\right\} \mid @_{l_{2}} \varphi} .
$$

Similarly for the other rules.

The second refinement attempts to replace branching rules by rules with fewer or no branches. For example, the rule for positive occurrences of $\square$,

$$
\frac{@_{l} \square_{a} \varphi, @_{l_{a}} \diamond_{a}\left\{l_{2}\right\}}{@_{l} \neg \diamond_{a}\left\{l_{2}\right\} \mid @_{l_{2}} \varphi}, \text { is refined to } \frac{@_{l} \square_{a} \varphi, @_{l} \diamond_{a}\left\{l_{2}\right\}}{@_{l_{2}} \varphi} .
$$

Other refined rules in the presented calculus are the rules expressing triangular properties in $(3 \mathrm{~g})$ and $(3 \mathrm{~h})$. These rule refinements are justified because the $(\dagger)$ condition from $[11\rfloor$ can be shown to hold in each case. The presented calculus is therefore sound and complete for IEL.

Finally, if the logic has the finite model property then the generated tableau calculus can be turned into a decision procedure by adding the blocking mechanism introduced in $\lfloor 9\rfloor$ which is based on the following unrestricted blocking rule:

$$
\text { (UB): } \frac{@_{l}\{l\}, @_{l_{0}}\left\{l_{0}\right\}}{@_{l}\left\{l_{0}\right\} \mid @_{l} \neg\left\{l_{0}\right\}}
$$

For the static part of IEL the finite model property is obtained by a standard filtration argument. The reduction axioms introduced before provide a way to translate formulae from the dynamic part to equivalent formulae in the static language. The translation is:

$$
\begin{aligned}
& t(p)=p, \quad t(\neg \varphi)=\neg t(\varphi), \quad t(\varphi \wedge \psi)=t(\varphi) \wedge t(\psi), \\
& t\left(\square_{a} \varphi\right)=\square_{a} t(\varphi) \quad \text { for } \square_{a} \in\left\{K_{a}, Q_{a}, X_{a}\right\}, \\
& t(\text { lhs })=t(r h s) \quad \text { for the reduction axioms in (1a)-(1d). }
\end{aligned}
$$

This implies that IEL has the finite model property and we can obtain the following result:

Theorem 1. The calculus listed on Figure 5 is sound and complete for IEL satisfiability and it is also terminating if equipped with the unrestricted blocking mechanism.

\section{Extension to Sequential Questioning Logic}

In this section we generalize the IEL/DELQ framework to a setting using questioning sequences and we call the emerging theory Sequential Questioning Logic (henceforth, $S Q L$ ).

The language of SQL is recursively defined by the following BNF:

$$
\varphi \quad::=\quad n|p| \neg \varphi|\varphi \vee \varphi| \square \varphi|[\sigma(k) ?] \varphi|[!] \varphi
$$

with $n, p, a, \neg, \vee, \square,[!]$ as before and $\sigma(n)$ ? representing dynamic questioning actions where $\sigma(n)=\left\langle\varphi_{0}, \ldots, \varphi_{n-1}\right\rangle$ is a sequence of SQL formulae. The semantics of the operators is also as before with the generalized intersection already introduced in Section 2 used for sequences. The fact that the questioning modalities are the only ones different between IEL and SQL dialects allows us to add questioning sequences while preserving all the other components. 
Tableau Synthesis for Interrogative Epistemic Logics S. Minică, M. Khodadadi, R. A. Schmidt, D. Tishkovsky

Figure 6: SQL specific dynamic connectives, semantics and tableau rules

\begin{tabular}{lcc}
\hline conn. type & semantics & rules \\
\hline & $\left.\forall x\left(\nu_{\mathrm{f}}([\sigma ?] \# p, x) \leftrightarrow \nu_{\mathrm{f}}(\# p, x)\right)\right)$ \\
& $\left.\forall x\left(\nu_{\mathrm{f}}([\sigma ?]\{n\}, x) \leftrightarrow \nu_{\mathrm{f}}(\{n\}, x)\right)\right)$ \\
& $\left.\forall x\left(\nu_{\mathrm{f}}([\sigma ?] \neg \varphi, x) \leftrightarrow \nu_{\mathrm{f}}(\neg[\sigma ?] \varphi, x)\right)\right)$ & \\
& As in Figure 5 with $[\sigma ?]$ instead of $[\varphi ?]$ \\
{$[\cdot ?] \quad[\mathrm{f}] \mapsto \boldsymbol{f}_{\forall x}\left(\nu_{\mathrm{f}}([\sigma ?](\varphi \wedge \psi), x) \leftrightarrow \nu_{\mathrm{f}}([\sigma ?] \varphi, x) \wedge \nu_{\mathrm{f}}([\sigma ?] \psi, x)\right)$} & \\
$\forall x\left(\nu_{\mathrm{f}}\left([\sigma ?] K_{a} \varphi, x\right) \leftrightarrow \nu_{\mathrm{f}}\left(K_{a}[\sigma], \varphi, x\right)\right)$ & \\
$\forall x\left(\nu_{\mathrm{f}}\left([\sigma(n) ?] Q_{a} \varphi, x\right) \leftrightarrow\right.$ see below & See the generalized rules below \\
$\forall x\left(\nu_{\mathrm{f}}\left([\sigma(n) ?] X_{a} \varphi, x\right) \leftrightarrow\right.$ see below & \\
\hline
\end{tabular}

The static part of SQL brings nothing new, it is axiomatized, as before in IEL, by standard hybrid logic axioms for nominals and intersection. The dynamic part of SQL brings some new features that generalize the initial setting from IEL, and we do not require formulae in $\sigma(n)$ to induce a partition of the domain.

The significant new feature in SQL relative to IEL is the presence of dynamic questioning modalities over sequences of questions. This has to bring about new reduction axioms. For formulae $\varphi$ with factual content the jump to questioning sequences is an obvious generalization of the pattern in previous reduction axioms. For such $\varphi_{0}, \varphi_{1}$ in a minimal sequence we have:

$$
\begin{aligned}
{\left[\varphi_{0}, \varphi_{1}\right] X \varphi \leftrightarrow } & \left(\varphi_{0} \wedge \varphi_{1} \wedge X\left(\left(\varphi_{0} \wedge \varphi_{1}\right) \rightarrow\left[\varphi_{0}, \varphi_{1}\right] \varphi\right)\right) \\
& \vee\left(\varphi_{0} \wedge \neg \varphi_{1} \wedge X\left(\left(\varphi_{0} \wedge \neg \varphi_{1}\right) \rightarrow\left[\varphi_{0}, \varphi_{1}\right] \varphi\right)\right) \\
& \vee\left(\neg \varphi_{0} \wedge \varphi_{1} \wedge X\left(\left(\neg \varphi_{0} \wedge \varphi_{1}\right) \rightarrow\left[\varphi_{0}, \varphi_{1}\right] \varphi\right)\right) \\
& \vee\left(\neg \varphi_{0} \wedge \neg \varphi_{1} \wedge X\left(\left(\neg \varphi_{0} \wedge \neg \varphi_{1}\right) \rightarrow\left[\varphi_{0}, \varphi_{1}\right] \varphi\right)\right) .
\end{aligned}
$$

This generalizes in the expected way to longer factual sequences. However, this cannot be extended beyond factual formulae, not even for minimal questioning sequences of length two. This approach fails for complex formulae that have questioning content or, in general, extrafactual or higher-order content. Consider as an illustration the following complex questioning formula: $\xi:=(\widehat{Q} i \rightarrow(j \vee k)) \wedge((\widehat{Q} j \wedge p) \rightarrow \widehat{Q} i)$. A model with a domain of three worlds $i, j, k$, universal issue and epistemic relations, and a valuation that makes $p$ true at $k$ provides a counterexample when we make the following substitutions: $\varphi_{0} \mapsto \xi, \varphi_{1} \mapsto \xi, \varphi \mapsto \neg p$.

For questioning sequences the disjunctive structure of the reduction axiom has to induce a partition of the domain. However, the questioning sequence does not have to give rise to a partition. This difference is not always fully understood and appreciated. If the questioning sequence has a more complex structure, for instance, if it induces a cover of the domain, more complex patterns are needed in the reduction axiom, that can ensure that the right hand side remains an exhaustive exclusive disjunction. In this way, fully general reduction axioms for SQL can be obtained and they will have the pattern given below.

We present the new additions in a synthetic way in Table 6 . The questioning modalities have a different type than before as they are now defined over lists of formulae. Except for this 
type difference most of the tableau rules will be as before. The new connectives using sequences will have new reduction axioms and new rules as specified in the table.

The reduction axioms will have the following pattern, for $\mathbf{\square} \in\{Q, X\}$ :

$$
[\sigma(n)] \mathbf{\square}_{a} \varphi \leftrightarrow \bigvee_{i=0}^{2^{|\sigma(n)|}}\left(\bigwedge_{k=0}^{|\sigma(n)|}\left([\sigma(k-1)] \varphi_{k}\right)^{\beta(i)(k)} \wedge \mathbf{\square}_{a}\left(\bigwedge_{k=0}^{|\sigma(n)|}\left([\sigma(k-1)] \varphi_{k}\right)^{\beta(i)(k)} \rightarrow[\sigma(n)] \varphi\right)\right),
$$

where $|\sigma(n)|$ is the length of the questioning sequence $\sigma(n)=\left\langle\varphi_{0}, . ., \varphi_{n-1}\right\rangle$,

and the value of $\varphi^{\beta(k)(i)}$ is determined by: $\varphi^{\beta(k)(i)}=\left\{\begin{aligned} \varphi & \text { if } \beta(k)(i)=1, \text { and } \\ \neg \varphi & \text { if } \beta(k)(i)=0 .\end{aligned}\right.$

$\beta(k)(i)$ represents the $i$-th position in the binary encoding $\beta(k)$ of the decimal number $k$.

The corresponding tableau rules are obtained as follows, for $\chi_{i}^{k}=\bigwedge_{k=0}^{|\sigma(n)|}\left([\sigma(k-1)] \varphi_{k}\right)^{\beta(i)(k)}$ :

$$
\frac{@_{l}[\sigma ?] \mathbf{\square}_{a} \varphi}{@_{l} \bigwedge_{k=0}^{|\sigma(n)|} \chi_{1}^{k} \wedge \mathbf{\square}_{a}\left(\bigwedge_{k=0}^{|\sigma(n)|} \chi_{1}^{k} \rightarrow[\sigma(n)] \varphi\right)|\cdots| @_{l} \bigwedge_{k=0}^{|\sigma(n)|} \chi_{n}^{k} \wedge \mathbf{\square}_{a}\left(\bigwedge_{k=0}^{|\sigma(n)|} \chi_{n}^{k} \rightarrow[\sigma(n)] \varphi\right)}
$$

In addition the complexity function for formulae has to add to Equation 2 values that take into account the length of the questioning sequences.

\section{Implementing an IEL Prover with MetTeL ${ }^{2}$}

In this section, we describe our experience in using MEтTeL 2 13 to generate a tableau prover for the tableau calculus derived in the previous section. METTEL ${ }^{2}$ is a prototypical tableau prover generator developed with the tableau synthesis framework as its theoretical foundation. Given the specification of a logic and the specification of a tableau calculus for this logic MeTTEL 2 generates JAVA code for a tableau prover implementing the tableau calculus. MetTeL $L^{2}$ has been successfully applied to several of logics, including Boolean logic, modal logics K, KT, S4, description logics $\mathcal{A} \mathcal{L C O}$ and $\mathcal{A L B O}$ id, and a hybrid logic with global counting operators 6 . The list is constantly growing. These test cases and downloadable copies of the generated provers are publicly available from the METTEL website.

The underlying language for syntax specification of IEL in MetTeL $\mathrm{L}^{2}$ is in line with the tableau synthesis framework object specification language. As the object language is settled in Section 3, preparing the syntax specification for METTEL2 is straightforward. For example, the syntax specification contains declaration of four sorts.

sort formula, agent, prop, individual;

Further, declarations of each connectives follow their representation in Figure 1. For instance, the connective \# is specified by the following declaration.

formula proposition = '\#' prop;

The declaration of the dynamic modality for questioning is given by:

formula query = '[?' formula ']' agent formula;

The syntax for Skolem terms which are fresh labels introduced during the application of diamond rules is given as follows. 


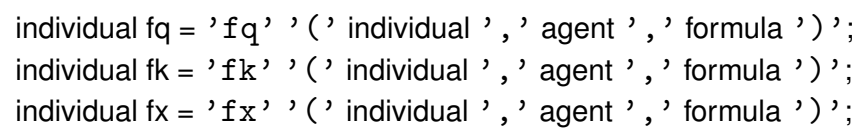

The specification of the tableau calculus in MetTeL 2 reflects all the rules of Figure 5 There are decomposition rules for positive as well as negative occurrences of all connectives. The exception is negation, which only has a rule for negative occurrence, namely elimination of double negation. For example, the rules for the three static modalities are specified as follows.

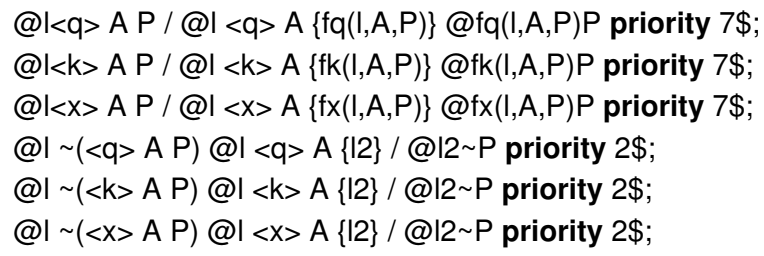

The rules for dynamic modalities have specific cases for atomic formulae, i.e., propositional atoms or nominals, and cases for complex formulae with non-factual content: questioning, epistemic or both. The cases for atomic formulae are specified as follows.

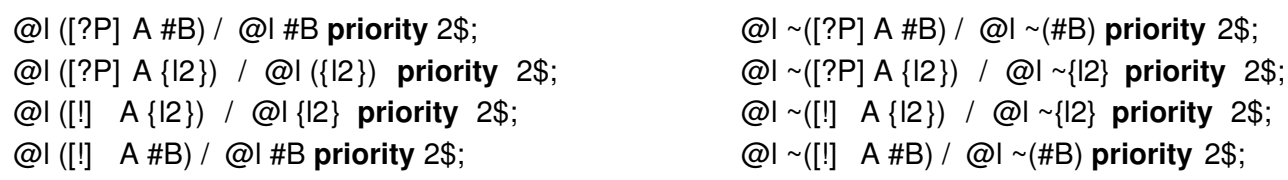

Having the rules for intersection of accessibility relations in the background theory is important because it plays a crucial role in the reduction rule for the dynamic modalities:

$@|<q>A\{12\} @|<k>A\{\mid 2\} / @ \mid<x>A\{\mid 2\}$ priority 2\$;

$@|<x>A\{\mid 2\} / @|<q>A\{\mid 2\} @ \mid<k>A\{\mid 2\}$ priority 2\$;

In MetTeL ${ }^{2}$, appearance of an equality formula in a branch immediately triggers ordered rewriting within the branch. The unrestricted blocking rule is implemented with use of this feature and the equality formula on its left branch forces the branch to be rewritten with respect to the additional equality.

An important feature provided by the MeTTeL implementation is the possibility to assign priorities to the tableau rules. This can be used to control the way the rules are applied in the generated prover. Each rule is followed by a number, which defines the rule application priority. Rules with smaller priority values have higher priority and applied more eagerly. Use of this feature is essential for the efficiency of the generated provers and especially in the case of IEL because the rules corresponding to reduction axioms with disjunctive patterns can be assigned lower priorities (higher priority values), thus reducing the branching factor and improving efficiency.

From the syntax specification and the tableau calculus, a tableau prover for IEL is automatically generated by METTEL ${ }^{2}$ according to the process described in detail in 13 . The generated prover can be used like most other tableau provers. Given a set of formulae as an input, the prover returns an answer Satisfiable or Unsatisfiable together with a model in the first case or with a set of contradictory formulae in the latter case.

We have tested the generated prover on a small set of sample formulae, where all the answers were correct. 


\section{Implementing IEL and SQL in Haskell}

The second implementation uses the literate Haskell script Qtab.lhs. In this section, we provide a brief description of this implementation and include the implementation itself in the long version of the paper 8 . The implementation started from a pre-existing tableau prover for hybrid logic 16」, to which we have added the details needed to model dynamic questioning actions. The tableau construction functionality was also completely changed. The current setting is more congenial with the framework from 11], which includes having a background theory for intersection and using unrestricted blocking.

We give next a broad view of the modules contained in the Qtab.Ihs architecture and their functionality. Syntax.hs: Preliminary module containing the data structures for modal and first-order logic formulae as well as the tableaux. Qtab.Ihs: The module containing the main functionality for the tableau prover such as the decide function that takes a formula in the IEL language and decides if it is or is not a tautology. Also functions controlling the order in which the formulae are analysed. Decomp.hs: The module containing the functionality associated with tableau expansion rules by logical decomposition. This proceeds either by standard logical analysis or by rules synthesized from reduction axioms. Backgrd.hs: The module containing the main components of the IEL background theory. In particular, the rules governing the behaviour of nominals and the rules for intersection are defined here. Also the unrestricted blocking mechanism is handled by functions in this module. Divide.hs: The order in which branches in a tableau are expanded is determined by their syntactic structure. The module contains functions used to recognize structural properties of formulae and to divide tableau nodes into component lists of prioritised formulae. Auxilar.hs: The module containing auxiliary functionality (such as displaying tableaux and translating formulae).

One particular aspect in which the Haskell implementation proved to be useful was in dealing with questioning sequences. Questioning sequences can also be added in METTEL 2 , see the rules for sequences of length two in the appendix of the long version. The features of functional programming and the way in which recursion is implicitly built in Haskell definitions makes working with arbitrary sequences of questions easier. It also made it obvious that modalities capturing questioning sequences can be modelled as fully functional algebraic data structures suitable for recursive manipulation. The decomposition rules for the static connectives and resolution are as in Figure 5. We include several illustrations of Qtab. Ihs output for questioning sequences of length two in the long version. The decomposition rules for the general case of arbitrarily long sequences follow the pattern of the rules from Table 6. We include below some illustrative examples of prover output for some paradigmatic examples of SQL formulae:

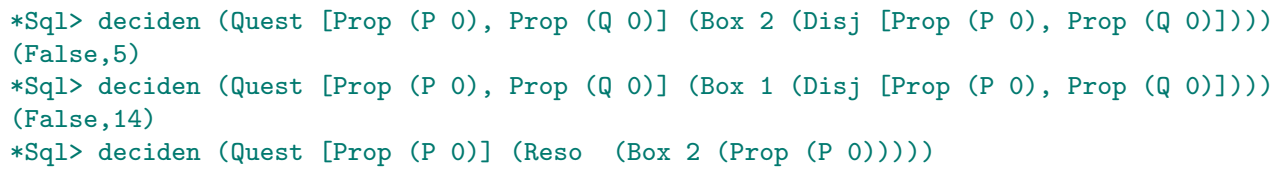

The first example illustrates a questioning sequence of length two combined with the static knowledge modality, which has a commutating behaviour. The second example illustrates a questioning sequence of length two in combination with the static issue modality, which uses reduction axioms based on the disjunction pattern: The third example illustrates a questioning sequence combining both asking actions and resolution actions. Because the resolution modality is idempotent, all resolution sequences are equivalent to a sequence of length one. Therefore the following reduction axioms are used for dealing with the aspect of the interaction between 
Tableau Synthesis for Interrogative Epistemic Logics S. Minică, M. Khodadadi, R. A. Schmidt, D. Tishkovsky

a questioning-sequence followed by a resolution-sequence:

$$
[\sigma ?][!] Q_{a} \psi \leftrightarrow[\sigma ?] Q_{a}[!] \psi, \quad[\sigma ?][!] K_{a} \psi \leftrightarrow[\sigma ?] X_{a}[!] \psi, \quad[\sigma ?][!] X_{a} \psi \leftrightarrow[\sigma ?] X_{a}[!] \psi
$$

The final illustration shows the difference between knowing that and knowing whether. After a yes/no question about $p$ the issue relation decides whether $p$, this turns out to be a SQL validity, however, it does not settle that $p$ holds.

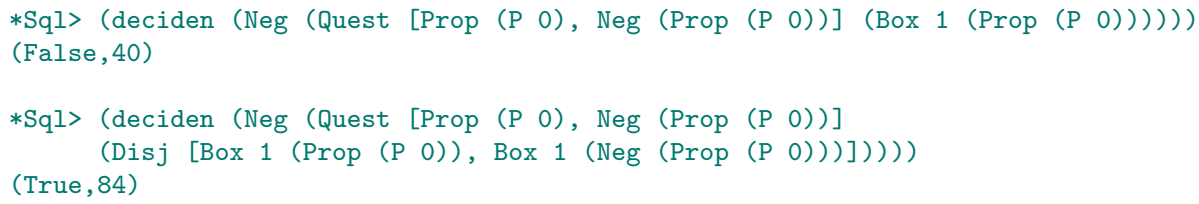

More detailed code output, traces of step-by step inference and tableau generation, and further explanation of the code functionality is included in the long version of the paper.

\section{Concluding Remarks}

In this paper we have shown what can be achieved when applying tableau synthesis and implementation for dynamic modalities of the simplest kind. This is only an initial illustration that serves as a case study for further extensions. We have considered one such extension to questioning sequences. Further extensions that we want to consider in the future include dynamic questioning actions that can model privacy and insecure communication and employ product update $[2,1]$ for computing issue relations $[7$, and a richer repertoire of questioning actions that go beyond the propositional case and include wh-questions $[5,18$.

MetTeL $L^{2}$ provides a robust and efficient platform for automatically generating a tableau prover for IEL. On the small set of formulae we used for testing, METTeL 2 was faster than the Haskell prover, because it implements clever backtracking techniques and other optimisations, currently not supported in the Haskell implementation.

On the other hand, the Haskell implementation provides a framework in which more experimental features of further extensions can be easily programmed and tested before they are ready to become mainstream conditions. We used the case of SQL to illustrate such an extension. We conclude with two main points about the overall significance of our approach.

We have shown how a dynamic component, in particular, dynamic questioning actions, can be integrated in the tableau synthesis framework. Based on the synthesised tableau calculus, two implementations have been developed: MeTTeL 2 and Qtab.lhs. This dynamic extension relies on rules in which the complexity of the formulae inside the scope of the dynamic modalities is reduced, even if the complexity of the conclusion formula in the rule can increase.

The second contribution facilitated by the implementations is an extension of the underlying dynamic logic itself. Implementing the reduction details made it obvious that a logical language containing sequences of questions, not just modalities for questioning actions, can be modelled in the framework, and extends the dynamic logic in a useful direction. 


\section{References}

[1] A. Baltag. Logics for insecure communication. In Proceedings of the 8th Conference on Theoretical Aspects of Rationality and Knowledge, pages 111-121. Morgan Kaufmann, 2001.

[2] A. Baltag, L. S. Moss, and S. Solecki. The logic of public announcements, common knowledge, and private suspicions. In Proceedings of the 7th Conference on Theoretical Aspects of Rationality and Knowledge, TARK '98, pages 43-56. Morgan Kaufmann, 1998.

[3] L. del Cerro, D. Fauthoux, O. Gasquet, A. Herzig, D. Longin, and F. Massacci. Lotrec: the generic tableau prover for modal and description logics. In Proceedings of the First International Joint Conference on Automated Reasoning, pages 453-458. Springer, 2001.

[4] H. Ditmarsch, W. Hoek, and B. Kooi. Dynamic epistemic logic. Springer, 2007.

[5] J. Hintikka, I. Halonen, and A. Mutanen. Interrogative logic as a general theory of reasoning. In D. M. Gabbay, R. H. Johnson, H. J. Ohlbach, and J. Woods, editors, Handbook of logic of argument and inference: The turn towards the practical, pages 295-337. Elsevier, 2002.

[6] M. Khodadadi, R. A. Schmidt, D. Tishkovsky, and M. Zawidzki. Terminating tableau calculi for modal logic K with global counting operators. Manuscript, http://www.mettel-prover.org/ papers/KEn12.pdf, 2012.

[7] Ş. Minică. Dynamic Logic of Questions. PhD thesis, ILLC, University of Amsterdam, 2011.

[8] Ş. Minică, M. Khodadadi, D. Tishkovsky, and R. A. Schmidt. Synthesising and implementing tableau calculi for interrogative epistemic logics, 2012. Long version of the present paper, http: //www.mettel-prover.org/papers/IEL-long.pdf.

[9] R. A. Schmidt and D. Tishkovsky. Using tableau to decide expressive description logics with role negation. In Proc. ISWC $2007+A S W C 2007$, volume 4825 of $L N C S$, pages 438-451. Springer, 2007.

[10] R. A. Schmidt and D. Tishkovsky. A general tableau method for deciding description logics, modal logics and related first-order fragments. In Proc. IJCAR 2008, volume 5195 of $L N C S$, pages 194-209. Springer, 2008.

[11] R. A. Schmidt and D. Tishkovsky. Automated synthesis of tableau calculi. Logical Methods in Computer Science, 7(2):1-32, 2011.

[12] B. D. ten Cate. Model Theory for Extended Modal Languages. PhD thesis, ILLC, University of Amsterdam, 2005.

[13] D. Tishkovsky, R. A. Schmidt, and M. Khodadadi. MetTeL2: Towards a tableau prover generation platform. In these proceedings, 2012.

[14] J. van Benthem. Logical dynamics of information and interaction. Cambridge Univ. Press, 2011.

[15] J. van Benthem and Ş. Minică. Toward a dynamic logic of questions. In Xiangdong He, John F. Horty, and Eric Pacuit, editors, Logic, Rationality, and Interaction, volume 5834 of Lecture Notes in Computer Science, pages 27-41. Springer, 2009.

[16] J. van Eijck. Hylotab: Tableau-based theorem proving for hybrid logics, 2002. Manuscript, CWI, Amsterdam.

[17] J. van Eijck. DEMO: A demo of epistemic modelling. In Interactive Logic. Selected Papers from the 7th Augustus de Morgan Workshop, London, volume 1, pages 303-362, 2007.

[18] A. Wiśniewski. Erotetic search scenarios, problem solving, and deduction. Logique E Analyse, 185-188:139-166, 2004. 